\title{
Padrões alimentares de adolescentes e associação com fatores de risco cardiovascular: uma revisão sistemática
}

\author{
Dietary habits of adolescents and associated cardiovascular \\ risk factors: a systematic review
}

David Franciole de Oliveira Silva ${ }^{1}$

Clélia de Oliveira Lyra ${ }^{1}$

Severina Carla Vieira Cunha Lima ${ }^{1}$

${ }^{1}$ Departamento de Nutrição, Universidade Federal do Rio Grande do Norte. Av. Senador Salgado Filho 3.000, Lagoa Nova. 59058-970 Natal RN Brasil. davfranci@hotmail.com
Abstract The scope of this study was to conduct a systematic review to establish the major dietary habits of adolescents and the corresponding association with cardiovascular risk factors. Research was performed in the LILACS, SciELO, PubMed, Scopus and Web of Science databases in addition to a manual search for original articles published between 2005 and 2014. Of the 371 entries identified; 26 fulfilled the inclusion criteria and were covered in the review. The main dietary habits observed were Western (61\%) Healthy (42\%) and Traditional (38\%). Western dietary habits were positively associated with total cholesterol and $S M$, while Healthy dietary habits were inversely associated with fasting glycaemia, DBP and SM and positively with $\mathrm{HDL}-\mathrm{C}$, all of the aforementioned associations with very low $(D)$ quality of evidence in accordance with the GRADE rating. The Traditional dietary habits were considered a risk factor for hyperglycemia, hyperinsulinemia and hypertriglyceridemia and protection for physical activity and eutrophic BMI. Although these results need to be analyzed with caution, due to the low quality of evidence, there is a clear need for actions aimed at promoting healthy dietary habits in adolescents in order to contribute to the prevention of cardiovascular risk factors.

Key words Food consumption, Dietary habit, Cardiovascular disease, Adolescent, Systematic review.
Resumo O objetivo deste estudo foi identificar por meio de uma revisão sistemática os principais padrões alimentares de adolescentes e verificar sua associação com fatores de risco cardiovascular. Foi realizada busca nas bases de dados Lilacs, SciELO, PubMed, Scopus e Web of Science, além da busca manual de artigos originais publicados entre 2005 a 2014. Dos 371 registros identificados; 26 preencheram os critérios de inclusão e foram inseridos na revisão. Os principais padrões alimentares observados foram Ocidental (61\%), Saudável (42\%) e Tradicionais (38\%). O padrão Ocidental foi positivamente associado com colesterol total e Sindrome Metabólica (SM), enquanto que o Saudável associou-se inversamente com glicemia de jejum, Pressão Arterial Diastólica (PAD) e SM e positivamente com $H D L$-c, todas estas associações com qualidade da evidência $D$, muito baixa, segundo o sistema GRADE. Os padrões Tradicionais se relacionaram como fator de risco para hiperglicemia, hiperinsulinemia e hipertrigliceridemia e proteção para atividade física e IMC eutrófico. Por mais que estes resultados precisem ser analisados com prudência, devido à baixa qualidade da evidência, observa-se a necessidade de medidas que visem à promoção da alimentação saudável em adolescentes, de modo a contribuir para a prevenção de fatores de risco cardiovascular.

Palavras-chave Consumo alimentar, Padrão alimentar, Doença cardiovascular, Adolescentes, Revisão sistemática 


\section{Introdução}

Monitorar o padrão de consumo alimentar e dietético de indivíduos ou grupos é uma importante ferramenta para identificar mudanças ou tendências de consumo, assim como também, para conhecer o papel da dieta como variável de exposição e sua relação com os desfechos. Ademais, pode ser utilizado como um indicador indireto do estado nutricional de uma população ${ }^{1-4}$. A utilização de informações referentes à dieta, e em especial as particularidades quanto às combinações de alimentos como as descritas por padrões alimentares, apresenta a vantagem de proporcionar a ampliação do conhecimento a respeito do papel que desempenha sobre determinados fatores de risco à saúde, considerando as interações que ocorrem entre os nutrientes ${ }^{1}$.

Para a identificação dos padrões alimentares utilizam-se vários métodos tanto de inquéritos alimentares quanto de análise de dados ${ }^{2,3,5}$. Dentre os métodos de inquéritos de avaliação do consumo alimentar e dietético destacam-se: Diário ou Registro Alimentar, História Alimentar, Recordatório Alimentar 24 horas (R24h) e Questionário de Frequência Alimentar (QFA) 3,5,6. Quanto à análise dos dados obtidos do consumo alimentar e dietético, os métodos se dividem em dois grupos: a priori e a posteriori ${ }^{2,4,7,8}$. Os métodos definidos a priori, por hipótese-orientada, são aqueles que utilizam escores ou índices dietéticos para avaliar a aderência de indivíduos a determinados guias ou recomendações dietéticas pré-estabelecidos ${ }^{2,4,7-9}$. Os métodos a posteriori consistem na definição dos padrões alimentares depois que os dados dietéticos são coletados ${ }^{7,8,10}$. Para isso fazem uso de análises estatísticas específicas, a saber: Análise Fatorial ${ }^{11,12}$, Análise de Agrupamentos $^{13,14}$ e Regressão por Redução de Posto $^{15,16}$.

Pesquisadores do campo da epidemiologia nutricional têm observado diversos padrões alimentares como o Ocidental, o Mediterrâneo, os considerados como Tradicionais de cada país e os Saudáveis. Cada um destes padrões apresentam associações distintas com características de estilo de vida, assim como também diferentes efeitos sobre a saúde humana $a^{7,17,18}$.

$\mathrm{Na}$ população adolescente, verifica-se que o padrão de consumo alimentar caracteriza-se pela baixa ingestão de frutas, legumes e verduras e pelo consumo excessivo de bebidas e alimentos industrializados e lanches do tipo fast-food ${ }^{19-22}$. Este comportamento alimentar é preocupante, visto que pode levar ao excesso de peso e a maior probabilidade de doenças crônicas não transmissíveis (DCNT) como câncer ${ }^{23}$, diabetes ${ }^{24}$, e morbidades e/ou fatores de risco para doenças cardiovasculares (DCV $)^{25}$.

A utilização de padrões alimentares constitui recurso de grande relevância para a identificação e a caracterização de comportamentos alimentares que se enquadram como inadequados. Neste sentido, pode permitir o desenvolvimento de estratégias que visem à mudança deste comportamento alimentar de risco, a fim de garantir o pleno potencial de crescimento e desenvolvimento dos adolescentes.

O objetivo deste estudo foi realizar uma revisão sistemática no que se refere aos padrões alimentares de adolescentes, e verificar a associação destes com fatores de risco para DCV.

\section{Métodos}

Realizou-se uma revisão sistemática de estudos sobre padrões alimentares de adolescentes do Brasil e de outros países. Utilizaram-se as recomendações do documento Preferred Reporting Items for Systematic Reviews and Meta-Analyses (PRISMA ${ }^{26}$, o qual tem como objetivo orientar a divulgação de revisões sistemáticas e meta-análises na área da saúde.

\section{Estratégia de busca}

A busca virtual foi realizada nas bases de dados Lilacs, SciELO, PubMed, Scopus e Web of Science, de artigos originais publicados entre 2005 e 2014. No PubMed, foi utilizada a seguinte estratégia de busca: (diet pattern OR diet patterns OR dietary pattern OR dietary patterns OR eating pattern OR eating patterns) AND (factor analysis OR principal component analysis OR cluster analysis OR reduced rank regression) AND (smoke OR cigarette OR cigarette smoking OR tobacco use OR sedentary $O R$ sedentariness $O R$ physical activity OR physical exercise OR body mass index $O R$ BMI OR excess weight OR overweight OR obesity $O R$ waist circumference OR abdominal obesity OR blood pressure OR hypertension OR dyslipidemia OR total cholesterol OR triglycerides $O R$ $H D L-c$ OR high-density lipoprotein OR LDL-c OR low density lipoprotein OR diabetes OR diabetes mellitus OR insulin resistance OR glycemia OR insulinemia OR metabolic syndrome) AND (adolescent OR adolescents). Nas bases Scopus e Web of Science, as estratégias de busca foram semelhantes a do PubMed. No Lilacs e no SciELO 
foi utilizada a seguinte combinação de termos: ("padrão alimentar" OR "padrões alimentares") AND ("análise fatorial” OR "análise de componentes principais” OR "regressão por redução de posto") AND (adolescente OR adolescentes). As pesquisas foram realizadas na primeira semana de outubro de 2014, sendo limitadas a artigos publicados entre janeiro 2005 e outubro de 2014, nos idiomas português, inglês ou espanhol.

\section{Critérios de inclusão e exclusão dos estudos}

Os estudos foram considerados elegíveis considerando os seguintes aspectos: 1) fossem artigos originais; 2) tivessem determinado os padrões alimentares por meio de métodos a posteriori, ou seja, Análise Fatorial, Análise de Agrupamentos ou Regressão por Redução de Posto; 3) tivessem avaliado exclusivamente adolescentes na faixa etária entre 10 a 19 anos e 11 meses; 4) avaliado a associação entre os padrões alimentares obtidos com quaisquer dos seguintes fatores de risco cardiovascular: tabagismo, prática de atividade física, estado nutricional antropométrico, pressão arterial, dislipidemias, alterações no metabolismo da glicose e/ou síndrome metabólica; e, 5) publicados em português, inglês ou espanhol. Os critérios de exclusão foram: dissertações, teses, e artigos de revisão da literatura.

Inicialmente, os títulos e os resumos dos artigos foram analisados a fim de verificar se atendiam aos critérios de inclusão definidos e/ou se apresentavam algum critério de exclusão. Quando houve dúvida quanto aos critérios de elegibilidade, todo o artigo foi analisado. Dois pesquisadores realizaram a análise dos estudos incluídos na revisão, sendo que, em caso de dúvida, um terceiro pesquisador foi consultado.

\section{Avaliação da qualidade metodológica dos estudos}

A avaliação da qualidade metodológica dos estudos incluídos foi realizada por meio das recomendações do Strengthening the Reporting of Observational Studies in Epidemiology Statement $(\mathrm{STROBE})^{27}$, o qual consiste em 22 itens que recebem pontuação de 0 a 1 . Depois da avaliação de todos os itens, os estudos receberam notas de 0 a 22. Com base nas notas recebidas, três categorias para avaliação da qualidade foram estabelecidas: A - quando o estudo preencher mais 80\% dos critérios estabelecidos no STROBE; B quando $50-80 \%$ dos critérios forem preenchidos; C - quando menos de $50 \%$ dos critérios forem preenchidos. Dois autores realizaram a avaliação quanto à qualidade metodológica dos estudos, sendo que, em caso de dúvida, um terceiro pesquisador foi consultado.

\section{Avaliação da qualidade da evidência}

Além da avaliação da qualidade metodológica dos estudos por meio do STROBE, procedeu-se à avaliação da qualidade da evidência pelo Sistema Grading of Recommendations Assessment, Development and Evaluation (GRADE) ${ }^{28,29}$, tendo em vista proporcionar maior confiabilidade à análise crítica dos achados. Neste estudo, realizamos a avaliação pelo sistema GRADE por desfecho, para obter o sumário da evidência, uma vez que a maioria dos estudos avaliou mais de um fator de risco para DCV. Para a avaliação dos estudos, foram utilizados os seguintes critérios: delineamento - ensaios clínicos partem de A e estudos observacionais de C; qualidade metodológica vieses conservadores, forte associação e consistência dos achados entre os estudos aumentam a nota e limitações metodológicas sérias, evidências indiretas e viés de publicação a diminuem. Após avaliados conforme o sistema GRADE, os estudos foram classificados em quatro categorias de qualidade da evidência: Alta - Há forte confiança de que o verdadeiro efeito/associação esteja próximo do estimado; Moderada - Há confiança moderada no efeito/associação estimado; Baixa - A confiança no efeito/associação é limitada; e, Muito Baixa - A confiança na estimativa do efeito/associação é muito limitada. Há importante grau de incerteza nos achados ${ }^{30}$.

\section{Síntese e comparação dos estudos}

O local do estudo foi descrito conforme o país e, quando disponível, segundo o estado e a cidade de realização. Quanto ao aspecto temporal, os artigos foram apresentados segundo o ano de publicação e, quando aplicável, o período de seguimento. A população de cada estudo foi caracterizada conforme o número e a faixa etária e/ou idade média dos participantes. Os métodos utilizados para obtenção dos dados de consumo alimentar e dietético dos participantes, assim como a técnica estatística utilizada para identificação dos padrões alimentares também foram apresentados. Os padrões alimentares obtidos nos estudos foram caracterizados conforme a denominação que receberam e os itens alimentares que os constituíam. Além disso, foram apresentadas as associações entre os padrões alimentares 
e os seguintes fatores de risco para DCV, quando disponíveis: tabagismo, atividade física, estado nutricional, pressão arterial, dislipidemias, alterações no metabolismo da glicose e síndrome metabólica.

\section{Resultados}

A busca eletrônica retornou 366 resultados, aos quais foram adicionados cinco artigos obtidos por meio da busca manual em referências de estudos e outras fontes. Destes 371 registros, 128 eram duplicados, os quais foram excluídos, resultando em 243 resumos selecionados para avaliação. Após leitura do título e do resumo foram excluídos 196 estudos. Depois de avaliados os critérios de inclusão e exclusão dos 47 artigos que foram lidos na íntegra, 26 estudos foram incluídos na revisão (Figura 1).

\section{Qualidade metodológica e características dos estudos}

Segundo os critérios STROBE ${ }^{27}$, dos 26 estudos incluídos na revisão, 14 foram de qualidade $\mathrm{A}$ (54\%) e 12 de qualidade B (46\%), não havendo, portanto, artigos de qualidade C. Foram obtidos estudos de países de várias regiões do mundo. $\mathrm{O}$ Brasil contribuiu com sete estudos ${ }^{31-37}$; Austrália contribuiu com cinco $^{38-42}$; Coréia do Sul, três ${ }^{43-45}$; China $^{46}$, Hong Kong ${ }^{47}$, Malásia ${ }^{48}$, EUA ${ }^{49}$, Irlan$\mathrm{da}^{50}$, Alemanha ${ }^{51}$, Nova Zelândia ${ }^{52}$, Ilhas Baleáricas (Espanha) ${ }^{53}$, Grécia ${ }^{54}$, México ${ }^{55}$ e Equador ${ }^{56}$ contribuíram com um cada. Os periódicos com maior número de artigos incluídos no estudo foram: Public Health Nutrition, com quatro artigos $^{38,46,53,54}$, British Journal of Nutrition ${ }^{40,43}$, The Journal of Nutrition ${ }^{42,49}$, Revista de Nutrição ${ }^{31,34} e$ Appetite ${ }^{33,50}$ com dois artigos cada. As outras revistas contribuíram com um estudo cada.

Nos artigos com adolescentes brasileiros a amostra estudada variou de $76^{31}$ a $1.139^{32}$ indivíduos. Já nos estudos com adolescentes de outros países o n amostral variou de $181^{49}$ a $5.003^{46}$. Em um dos estudos brasileiros, todos os participantes tinham obesidade ${ }^{31}$. Em quatro ${ }^{38-41}$ dos cinco $^{38-42}$ estudos obtidos entre adolescentes da Austrália, a população estudada foi a mesma, os participantes do Estudo Raine, uma coorte deste país. O que diferiu entre os estudos foi o tamanho da amostra, os objetivos a serem analisados e os respectivos desfechos. No presente estudo decidiu-se manter todos os artigos, tendo em vista que a exclusão de algum destes que utilizaram a

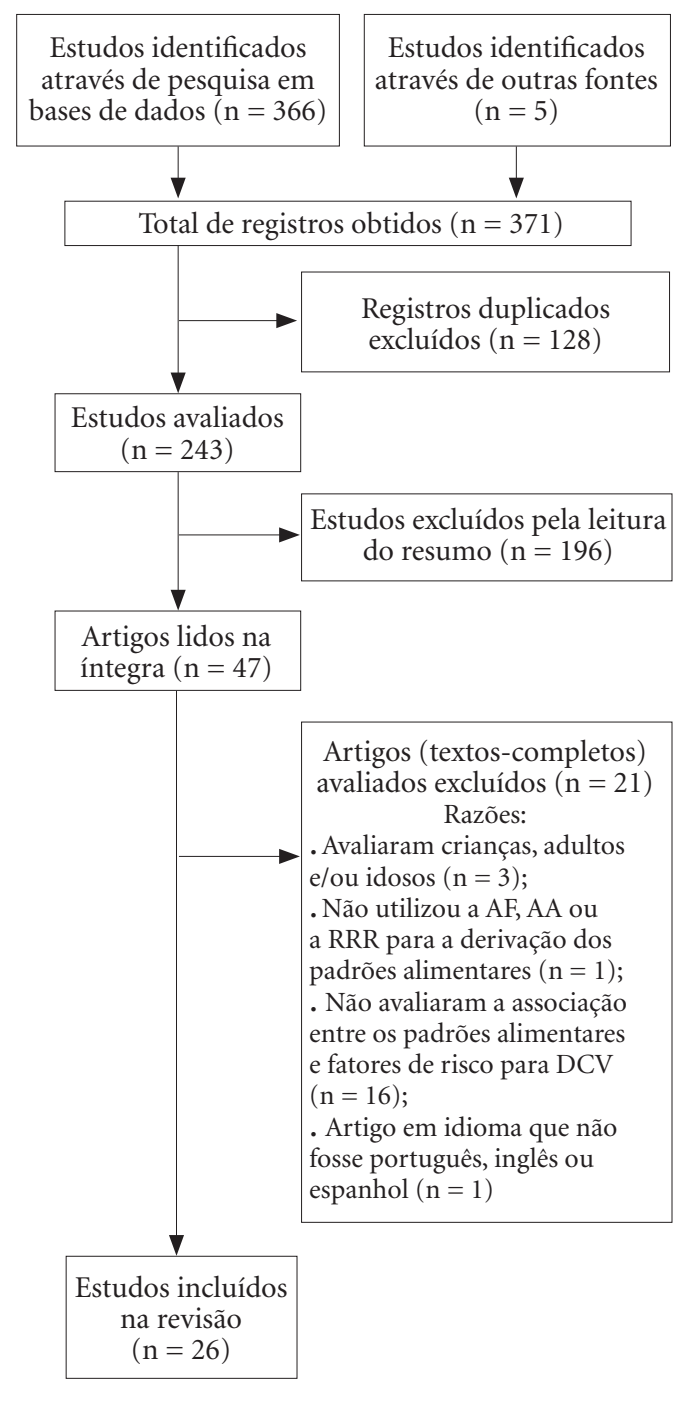

Figura 1. Fluxograma de seleção dos estudos.

Legenda: AF: Análise Fatorial; AA: Análise de Agrupamentos; RRR: Regressão por Redução de Posto.

mesma base de dados poderia levar à perda de resultados de avaliações pertinentes aos objetivos da presente revisão. A Tabela 1 apresenta as características dos estudos incluídos na revisão.

\section{Métodos utilizados e Padrões alimentares obtidos}

Em 15 estudos, o Questionário de Frequência Alimentar foi o método de inquérito alimentar utilizado $^{32,33,36-39,41,42,46-50,52,54}$; em cinco o Recordatório Alimentar ${ }^{35,44,45,55,56}$; três utilizaram o 
Tabela 1. Características dos estudos incluídos na revisão sistemática.

\begin{tabular}{|c|c|c|c|c|c|c|}
\hline Autor & Ano & País & $\mathrm{No}^{\circ}$ participantes & $\begin{array}{l}\text { Faixa } \\
\text { etária }\end{array}$ & $\begin{array}{c}\text { Pontuação } \\
\text { STROBE }\end{array}$ & $\begin{array}{l}\text { Qualidade } \\
\text { do estudo } \\
\text { (STROBE) }\end{array}$ \\
\hline Dishchekenian et al. ${ }^{31}$ & 2011 & Brasil & 76 (com obesidade) & 14 a 19 anos & 18,5 & A \\
\hline Rodrigues et al. ${ }^{32}$ & 2012 & Brasil & 1.139 & 14 a 19 anos & 18,5 & $\mathrm{~A}$ \\
\hline Moraes et al. ${ }^{33}$ & 2012 & Brasil & 991 & 14 a 18 anos & 19,5 & $\mathrm{~A}$ \\
\hline Salvatti et al. ${ }^{34}$ & 2011 & Brasil & 239 & 14 a 19 anos & 16,5 & $\mathrm{~B}$ \\
\hline Morais et al. ${ }^{35}$ & 2013 & Brasil & 430 & 10 a 19 anos & 18,0 & $\mathrm{~A}$ \\
\hline Pinho et al. ${ }^{36}$ & 2014 & Brasil & 474 & 11 a 17 anos & 18,0 & A \\
\hline Borges et al. ${ }^{37}$ & 2012 & Brasil & $\begin{array}{l}372 \text { no período } 1 \text {, e } \\
268 \text { no período } 2\end{array}$ & 10 a 15 anos & 18,0 & A \\
\hline Ambrosini et al..$^{38}$ & 2009 & Austrália & 1.613 & 14 anos & 17,5 & $\mathrm{~B}$ \\
\hline Ambrosini et al..$^{39}$ & 2010 & Austrália & 1.139 & 14 anos & 17,0 & $\mathrm{~B}$ \\
\hline Ambrosini et al..$^{40}$ & 2011 & Austrália & 783 & 14 anos & 15,5 & $\mathrm{~B}$ \\
\hline Oddy et al. ${ }^{41}$ & 2013 & Austrália & 995 & Aos 14 e 17 anos & 16,5 & $\mathrm{~B}$ \\
\hline McNaughton et al. ${ }^{42}$ & 2008 & Austrália & 764 & 12 a 18 anos & 16,5 & $\mathrm{~B}$ \\
\hline Song et al..$^{43}$ & 2005 & Coréia do Sul & 671 & 12 a 14 anos & 16,0 & $\mathrm{~B}$ \\
\hline Song et al. ${ }^{44}$ & 2010 & Coréia do Sul & 4.324 & 10 a 19 anos & 16,0 & $\mathrm{~B}$ \\
\hline Joug et al. ${ }^{45}$ & 2012 & Coréia do Sul & 3.168 & 13 a 18 anos & 13,5 & $\mathrm{~B}$ \\
\hline Weng et al. ${ }^{46}$ & 2011 & China & 5.003 & 11 a 16 anos & 18,5 & A \\
\hline Chan et al. ${ }^{47}$ & 2014 & Hong Kong & 351 & 10 a 12 anos & 18,5 & $\mathrm{~A}$ \\
\hline Garba et al. ${ }^{48}$ & 2014 & Malásia & 2.248 & 14 anos & 16,5 & $\mathrm{~B}$ \\
\hline $\mathrm{Li}$; Wang ${ }^{49}$ & 2008 & Estados Unidos & 181 & 10 a 14 anos & 17,5 & $\mathrm{~B}$ \\
\hline Fitzgerald et al. ${ }^{50}$ & 2013 & Irlanda & 483 & 13 a 18 anos & 15,0 & $\mathrm{~B}$ \\
\hline Richter et al..$^{51}$ & 2012 & Alemanha & 1.272 & 12 a 17 anos & 19,0 & A \\
\hline Howe et al..$^{52}$ & 2013 & Nova Zelândia & 681 & 14 a 18 anos & 18,0 & $\mathrm{~A}$ \\
\hline del Mar Bibiloni et al. ${ }^{53}$ & 2011 & Ilhas Baleáricas (Espanha) & 1.231 & 12 a 17 anos & 20,0 & A \\
\hline Kourlaba et al..$^{54}$ & 2009 & Grécia & 2.118 & 12 a 17 anos & 15,5 & $\mathrm{~B}$ \\
\hline Lozada et al..$^{55}$ & 2007 & México & 447 & 12 a 19 anos & 14,5 & $\mathrm{~B}$ \\
\hline Ochoa-Avilés et al. ${ }^{56}$ & 2014 & Equador & 779 & 10 a 16 anos & 19,5 & A \\
\hline
\end{tabular}

Registro alimentar ${ }^{31,34,43}$; um utilizou a História alimentar ${ }^{51}$; e dois o QFA aliado a outro tipo de inquérito, sendo que em um foi o Recordatório alimentar 24 horas $^{53}$, e em outro, o Registro alimentar ${ }^{40}$.

A análise fatorial foi o método utilizado para a derivação dos padrões alimentares em 22 estudos $^{31-42,46-54,56}$; três empregaram a análise de agrupamentos ${ }^{43-45}$ e um utilizou tanto a análise fatorial quanto a análise de agrupamentos para a derivação dos padrões alimentares ${ }^{55}$.

O número de padrões alimentares obtidos em cada estudo foi variado. Oito estudos obtiveram apenas dois padrões ${ }^{38-41,43,50,53,56}$, um identificou quatro ${ }^{34}$ e outro obteve sete ${ }^{54}$. A maior parte dos estudos, dezesseis, identificou três padrões alimentares $^{31-33,35-37,42,44-49,51,52,55}$.

A maioria dos estudos obteve um padrão alimentar considerado como não saudável, sendo que em 10 foi denominado de "Ocidental"32,38-41,44,49,51,53,55 (38\%), em quatro de "Junk
Food"33,34,36,54 (15\%), em um de "Fast Food"31 (4\%) e em outro de padrão "Não Saudável"50 (4\%). Em 11 estudos (42\%) obtiveram-se padrões alimentares classificados como "Saudáveis" 33,34,36,38-42,50,51,54; em 10 como sendo o "Tradicional" do país ${ }^{31,32,34-37,43,44,46,51}(38 \%)$ e em dois estudos como estilo "Mediterrâneo" ${ }^{33,54}$ (8\%).

\section{Associação entre padrões alimentares e tabagismo}

Dos estudos incluídos na revisão, três avaliaram a associação entre os padrões alimentares obtidos com o tabagismo ${ }^{32,38,54}$. Os estudos que verificaram associações estatisticamente significativas entre os padrões alimentares e os fatores de risco para DCV, incluindo os resultados estatísticos, estão detalhados no Quadro 1. No estudo realizado por Kourlaba et al. ${ }^{54}$, o padrão Junk Food foi positivamente associado com o tabagismo, e o padrão Vegetariano Saudável foi inver- 
Quadro 1.Estudos que avaliaram a associação entre padrões alimentares e fatores de risco para DCV em adolescentes*.

\begin{tabular}{|c|c|c|}
\hline Estudo & Padrões alimentares identificados & Principais Resultados \\
\hline \multicolumn{3}{|l|}{ Tabagismo } \\
\hline Kourlaba et al., $2009^{54}$ & $\begin{array}{l}\text { Padrão Junk food, Padrão Carne } \\
\text { Vermelha, Padrão Vegetariano } \\
\text { Saudável, Padrão Produtos } \\
\text { Lácteos, Padrão Massa e } \\
\text { Pão Integral, Padrão Dieta } \\
\text { Mediterrânea, Padrão Alimentos } \\
\text { Tradicionais da Cozinha Grega e } \\
\text { Padrão Ovos e Pão Branco. }\end{array}$ & $\begin{array}{l}\text { Padrão Junk food: }(+) \text { tabagismo }(\beta=0.88 \\
\text { CI95\% }=0.61-1.17 ; \mathrm{p}<0,001) \\
\text { Padrão Vegetariano Saudável: }(-) \text { tabagismo }(\beta \\
=-0.12 ; \text { CI } 95 \%=-0.38-0,14 ; \mathrm{p}=0.373)\end{array}$ \\
\hline \multicolumn{3}{|l|}{ Nível de atividade física } \\
\hline Richter et al., $2012^{51}$ & $\begin{array}{l}\text { (M): Padrão Ocidental, Padrão } \\
\text { Saudável e Padrão Tradicional. } \\
\text { (F): Padrão Ocidental e } \\
\text { Tradicional e Padrão Saudável. }\end{array}$ & $\begin{array}{l}\text { Padrão Tradicional: }(+) \text { nível de atividade física } \\
(\mathrm{M})(\mathrm{p}=0.042) . \\
\text { Padrão Saudável: }(+) \text { nível de atividade física ( } \mathrm{p} \\
=0.0398) .\end{array}$ \\
\hline $\begin{array}{l}\text { McNaughton et al., } \\
2008^{42}\end{array}$ & $\begin{array}{l}\text { Padrão Frutas, Salada, Cereais } \\
\text { \& peixe, Padrão Elevado Teor } \\
\text { de Gordura e Açúcar e Padrão } \\
\text { Vegetais }\end{array}$ & $\begin{array}{l}\text { Padrão Elevado Teor de Gordura e Açúcar } \\
\text { (estilo ocidental): }(+) \text { com atividade física (p } \\
<0.05) \text {. }\end{array}$ \\
\hline Moraes et al., 2012 33 & $\begin{array}{l}\text { Padrão Junk food, Padrão Saudável } \\
\text { e Padrão Proteína }\end{array}$ & $\begin{array}{l}\text { Padrão Saudável: }(+) \text { com atividade física }(\beta= \\
0.04 ; \text { CI } 95 \%=0.03-0.05 ; \mathrm{p}<0.0001)\end{array}$ \\
\hline $\begin{array}{l}\text { Rodrigues et al., } \\
2012^{32}\end{array}$ & $\begin{array}{l}\text { Padrão Ocidental, Padrão } \\
\text { Tradicional e Padrão Misto }\end{array}$ & $\begin{array}{l}\text { Padrão Misto: }(+) \text { física (sedentário/ } \\
\text { insuficiente sedentário/ativo) }(\beta=0,10 \text {; CI95\% } \\
=0,02-0,19 \text { e } \mathrm{p}=0,02) \text {. }\end{array}$ \\
\hline
\end{tabular}

continua

samente associado com o tabagismo. Nos outros estudos, não se verificou associação significativa entre os padrões alimentares e o tabagismo ${ }^{32,38}$. Estes resultados demonstram que, na presente revisão, a associação entre os padrões alimentares e o tabagismo foi inconclusiva. O Quadro 2 apresenta o sumário da evidência, incluindo a avaliação da sua qualidade, para a associação entre os padrões alimentares Ocidental e Saudável e os fatores de risco para DCV.

\section{Associação entre padrões alimentares e atividade física}

Avaliou-se a associação entre os padrões alimentares e a atividade física em oito estu$\operatorname{dos}^{32,33,38,42,49,51,53,54}$. Os resultados quanto aos padrões alimentares estilo Ocidental e a prática de atividade física revelaram que em um estudo houve associação positiva e significativa ${ }^{42}$, e, em outros sete, não foi verificada associação estatisticamente significativa ${ }^{32,33,38,49,51,53,54}$. Assim, o sumário da evidência é de que não há associação significativa entre o padrão Ocidental e a atividade física, com classificação GRADE C, baixa. Os padrões classificados como "Saudáveis" foram positivamente associados com atividade física em dois estudos ${ }^{33,51}$ e em outros três não se verificou associação estatisticamente significativa ${ }^{38,42,54}$, sendo o resultado inconclusivo. Em relação aos padrões Tradicionais de cada país, os identificados no Brasil e na Alemanha foram positivamente associados com atividade física ${ }^{32,51}$. Em estudo que avaliou a associação entre o padrão Mediterrâneo e a atividade física não foi verificada nenhuma associação ${ }^{53}$. 


\begin{tabular}{|c|c|c|}
\hline \multicolumn{3}{|l|}{ Quadro 1. continuação } \\
\hline Estudo & Padrões alimentares identificados & Principais Resultados \\
\hline \multicolumn{3}{|c|}{ Estado nutricional antropométrico e alterações metabólicas } \\
\hline $\begin{array}{l}\text { Dishchekenian et al., } \\
2011^{31}\end{array}$ & $\begin{array}{l}\text { Padrão Tradicional, Padrão Em } \\
\text { Transição e Padrão Junk food. }\end{array}$ & $\begin{array}{l}\text { Padrão Fast Food: }(+) \text { insulina }(\beta \text { padr }=0,170 ; \\
\mathrm{p}<0,001) \text {, colesterol }(\beta \text { padr }=0,243 ; \mathrm{p}= \\
0,001), \text { LDL-c }(\beta \text { padr }=0,314 ; \mathrm{p}=<0,001), \\
\text { PAS }(\beta \text { padr }=0,552 ; \mathrm{p}<0,001) \text { e PAD }(\beta \text { padr } \\
=0,504 ; \mathrm{p}<0,001), \text { e }(-) \text { HDL-c }(\beta \text { padr }= \\
-0,311 ; \mathrm{p}=<0,001) \text {. } \\
\text { Padrão Tradicional: }(+) \text { glicemia }(\beta \text { padr }= \\
0,425 ; \mathrm{p}<0,001) \text {, insulina }(\beta \text { padr }=0,304 ; \mathrm{p}< \\
0,001), \text { TG }(\beta \text { padr }=0,577 ; \mathrm{p}<0,001), \quad \text { e }(-) \\
\text { HDL-c }(\beta \text { padr }=0,270 ; \mathrm{p}=0,023) .\end{array}$ \\
\hline $\begin{array}{l}\text { Ambrosini et al., } \\
2010^{39}\end{array}$ & Padrão Saudável e Ocidental. & $\begin{array}{l}\text { Sexo feminino: } \\
\text { Padrão Saudável: }(-) \text { glicose sérica }(\mathrm{p}=0,04) \text {. } \\
\text { Padrão Ocidental: }(+) \text { elevado risco metabólico } \\
\text { vs. baixo risco metabólico - definido com base } \\
\text { em valores de IMC, PAS, TG e HOMA (OR = } \\
\text { 1,82; CI95\% }=1,28-2,60 ; \mathrm{p}=0.01) ;(+) \text { IMC (p } \\
=0,02) \text {, CC }(\mathrm{p}=0,03) \text { e CT }(\mathrm{p}=0,03) . \\
\text { Sexo masculino: } \\
\text { Padrão Saudável: }(-) \text { glicose }(\mathrm{p}=0,01),(+) \\
\text { HDL-c }(\mathrm{p}=0,02) .\end{array}$ \\
\hline $\begin{array}{l}\text { Ambrosini et al., } \\
2011^{40}\end{array}$ & Padrão Saudável e Ocidental. & $\begin{array}{l}\text { Padrão Saudável: }(-) \text { glicemia de jejum }(\mathrm{r}= \\
-0,09 ; \mathrm{p}<0.01) .\end{array}$ \\
\hline Salvatti et al., $2011^{34}$ & $\begin{array}{l}\text { Padrão Tradicional, Padrão } \\
\text { Urbano, Padrão Saudável e Padrão } \\
\text { Junk food }\end{array}$ & $\begin{array}{l}\text { Padrão Saudável: }(+) \text { sobrepeso. } \\
\text { Padrão Junk food: }(+) \text { sobrepeso. } \\
\text { Padrão Tradicional: }(+) \text { eutrofia e obesidade. }\end{array}$ \\
\hline Moraes et al., $2012^{33}$ & $\begin{array}{l}\text { Padrão Junk food, Padrão Saudável } \\
\text { e Padrão Proteína. }\end{array}$ & $\begin{array}{l}\text { Padrão Junk food: }(-) \text { CC }(\mathrm{M})(\beta=-0,18 \\
\text { CI95\% }=-0,03,-0,01 ; \mathrm{p}=0,003) .\end{array}$ \\
\hline Song et al., $2010^{44}$ & $\begin{array}{l}\text { Padrão alimentar Tradicional, } \\
\text { Padrão Alimentar Ocidental e } \\
\text { Padrão Alimentar Modificado. }\end{array}$ & $\begin{array}{l}\text { Padrão Ocidental: }(+) \uparrow \mathrm{TG}(\mathrm{M})(\mathrm{OR}=1,33 ; \\
\text { CI95\% }=0,97 \text { a } 1,83, \mathrm{p}=0,07),(+) \text { sobrepeso } \\
(\mathrm{M})(\mathrm{OR}=1,40 ; \mathrm{CI} 95 \%=1,00 \text { a } 1,97, \mathrm{p}=0,05)\end{array}$ \\
\hline $\begin{array}{l}\text { McNaughton et al., } \\
2008^{42}\end{array}$ & $\begin{array}{l}\text { Padrão Frutas, Salada, Cereais } \\
\text { \& peixe, Padrão Elevado Teor } \\
\text { de Gordura e Açúcar e Padrão } \\
\text { Vegetais. }\end{array}$ & $\begin{array}{l}\text { Padrão "Fruta, Salada, Cereais e Peixe" } \\
\text { (Saudável): }(-) \text { PAD em adolescentes com idade } \\
\geq 16 \text { anos }(\mathrm{p}=0,025) \text {. }\end{array}$ \\
\hline $\begin{array}{l}\text { Rodrigues et al., } \\
2012^{32}\end{array}$ & $\begin{array}{l}\text { Padrão Ocidental, Padrão } \\
\text { Tradicional e Padrão Misto. }\end{array}$ & $\begin{array}{l}\text { Padrão Tradicional: }(+) \text { IMC eutrófico }[\mathrm{sem} \\
\text { excesso de peso (categoria de referência) } / \text { com } \\
\text { excesso de peso }(B=-0,40 ; \text { CI } 95 \%=-0,55-0,56 \text {; } \\
p<0,01 \text {. }\end{array}$ \\
\hline Lozada et al., $2007^{55}$ & $\begin{array}{l}\text { Padrão Urbano-Pobre, Padrão } \\
\text { Rural e Padrão Ocidental. }\end{array}$ & $\begin{array}{l}\text { Padrão Urbano-Pobre: }(+) \text { sobrepeso }(\mathrm{RP}= \\
\text { 1.80; CI95\% }=1.00-3.24) \text {. Associação verificada } \\
\text { em relação ao Padrão Rural. } \\
\text { Padrão Ocidental: }(+) \text { Obesidade\#. }\end{array}$ \\
\hline Oddy et al., $2013^{41}$ & $\begin{array}{l}\text { Padrão Ocidental e Padrão } \\
\text { Saudável. }\end{array}$ & $\begin{array}{l}\text { Padrão Ocidental: }(+) \uparrow \operatorname{IMC}(\beta=1.41 \text {; CI95 \% } \\
=1.12-1.82 ; \mathrm{p}<0.0005)\end{array}$ \\
\hline
\end{tabular}




\begin{tabular}{|c|c|c|}
\hline \multicolumn{3}{|l|}{ Quadro 1. continuação } \\
\hline Estudo & Padrões alimentares identificados & Principais Resultados \\
\hline \multicolumn{3}{|c|}{ Estado nutricional antropométrico e alterações metabólicas } \\
\hline $\begin{array}{l}\text { del Mar Bibiloni et al., } \\
2011^{53}\end{array}$ & $\begin{array}{l}\text { Padrão Ocidental e Padrão } \\
\text { Mediterrâneo. }\end{array}$ & $\begin{array}{l}\text { Padrão Ocidental: }(-) \text { sobrepeso/obesidade }(\mathrm{M}) \\
(\mathrm{OR}=2.26 ; \mathrm{CI} 95 \%=1.04-4.88 ; \mathrm{p}=0,037)\end{array}$ \\
\hline Weng et al., $2011^{46}$ & $\begin{array}{l}\text { Padrão Lanche, Padrão Alimentos } \\
\text { animais e Padrão Tradicional. }\end{array}$ & $\begin{array}{l}\text { Padrão Lanche e Padrão Animal: (+) IMC } \\
\text { eutrófico }(p<0.001) .\end{array}$ \\
\hline Howe et al., $2013^{52}$ & $\begin{array}{l}\text { Padrão Guloseimas, Padrão Frutas } \\
\text { e Vegetais e Padrão Alimentos } \\
\text { Básicos. }\end{array}$ & $\begin{array}{l}\text { Padrão Alimentos Básicos: }(+) \text { CC }(\mathrm{M})(\beta= \\
-1.17 ; \text { CI95\% }=-1.96,-0.39 ; \mathrm{p}<0.05) .\end{array}$ \\
\hline Pinho et al., $2014^{36}$ & $\begin{array}{l}\text { Padrão Junk food, Padrão Saudável } \\
\text { e Padrão Tradicional. }\end{array}$ & $\begin{array}{l}\text { Padrão Saudável: }(-) \text { sobrepeso }(\mathrm{OR}=0.56 \\
\text { CI95\% }=0.35-0.91 ; \mathrm{p}<0.05)\end{array}$ \\
\hline $\begin{array}{l}\text { Ochoa-Avilés et al., } \\
2014^{56}\end{array}$ & $\begin{array}{l}\text { Padrão Rico em Arroz e Gordura } \\
\text { não Animal e Padrão Denso em } \\
\text { Trigo e Gordura Animal. }\end{array}$ & $\begin{array}{l}\text { Padrão Denso em Trigo e Gordura Animal: } \\
(+) \text { colesterol total }(\mathrm{p}=0.02) \text { e } \mathrm{LDL}(\mathrm{p}=0.04) \\
\text { entre os adolescentes que residiam em áreas } \\
\text { rurais. }\end{array}$ \\
\hline Li; Wang, $2008^{49}$ & $\begin{array}{l}\text { Padrão Ocidental, Padrão Oriental } \\
\text { e Padrão lácteos. }\end{array}$ & $\begin{array}{l}\text { Padrão Rico em Arroz e Gordura não Animal: } \\
(+) \uparrow \text { glicose em adolescentes que residiam em } \\
\text { áreas urbanas }(\mathrm{p}<0.01) \text {. } \\
\text { Adolescentes com sobrepeso foram }(+) \\
\text { associados com a diminuição do consumo de } \\
\text { alimentos do Padrão Ocidental em } 1 \text { ano de } \\
\text { seguimento (sobrepeso versus eutrofia) (OR = } \\
\text { - 0,37; CI95\% }=0.15,0.92, \mathrm{p}<0.05) \text {. }\end{array}$ \\
\hline Borges et al., $2012^{37}$ & $\begin{array}{l}\text { Padrão Tendência Multigrupo } \\
\text { (Tradicional brasileiro), Padrão } \\
\text { tendência HIDE (elevada } \\
\text { densidade energética) e Padrão } \\
\text { Tendência de Mudança. }\end{array}$ & $\begin{array}{l}\text { Padrão Tendência Multigrupo ( similar ao } \\
\text { Padrão Tradicional brasileiro): }(+) \text { baixo risco } \\
\text { de sobrepeso }(\mathrm{M}) \text { (tomando os resultados do } \\
\text { quintil } 5 \text { em relação ao quintil } 1(\mathrm{RR}=0.51 ; \mathrm{CI} \\
95 \%=0.28-0.95 ; \mathrm{p}<0.05) .\end{array}$ \\
\hline
\end{tabular}

Legenda: CC - Circunferência da Cintura; F - sexo feminino; HDL - Lipoproteína da Alta Densidade-c; HOMA - Modelo de Avaliação da Homeostase; IMC: Índice de Massa Corporal; LDL-c - Lipoproteína de Baixa Densidade-c; M - sexo masculino; PAD - Pressão Arterial Diastólica; PAS- Pressão Arterial Sistólica; TG - Triglicerídeos. * No Quadro 1 são mostrados apenas os dados referentes aos estudos que verificaram associação significativa entre os padrões alimentares obtidos e fatores de risco para DCV. \#Os dados estatísticos não foram apresentados.

\section{Associações entre os padrões alimentares e o estado nutricional antropométrico e as alterações metabólicas}

A associação entre os padrões alimentares e morbidades e/ou fatores de risco para DCV foram avaliados em 25 estudos $^{31-53,55,56}$. Padrões estilo "Ocidental" foram positivamente associados com IMC elevado em cinco estudos ${ }^{34,39,41,44,55}$, e em dois inversamente ${ }^{49,53}$. Para a circunferência da cintura, em um estudo verificou-se associação positiva $^{39}$ e em outro inversa ${ }^{33}$. Com relação ao metabolismo lipídico, dois estudos observaram associação positiva entre padrões estilo Ocidental e colesterol total ${ }^{31,39}$, um com triglicerídeo ${ }^{44}$, e um com LDL-c ${ }^{31}$. O estudo realizado por Dishchekenian et al. ${ }^{31}$, o qual foi realizado com adolescentes com obesidade, foi o único que observou associação positiva entre o padrão Ocidental com pressão arterial e insulina, e inversa com HDL-c. Com relação à síndrome metabólica, um estudo verificou associação positiva ${ }^{39}$. 
Quadro 2. Relação entre os padrões alimentares de adolescentes e fatores de risco cardiovascular com classificação da qualidade da evidência segundo o sistema GRADE.

\begin{tabular}{|c|c|c|c|c|c|}
\hline $\begin{array}{c}\text { Fator } \\
\text { de risco }\end{array}$ & $\begin{array}{c}\text { Padrão } \\
\text { Alimentar }\end{array}$ & $\begin{array}{l}\text { Associação } \\
\text { positiva }\end{array}$ & $\begin{array}{l}\text { Associação } \\
\text { negativa }\end{array}$ & $\begin{array}{c}\text { Sem } \\
\text { associação }\end{array}$ & $\begin{array}{c}\text { Sumário da } \\
\text { evidência (GRADE) }\end{array}$ \\
\hline Tabagismo & $\begin{array}{l}\text { Padrão } \\
\text { Ocidental }\end{array}$ & $\begin{array}{l}\text { Kourlaba et al., } \\
2009^{54}\end{array}$ & - & $\begin{array}{l}\text { Rodrigues et al., } 2012^{32} \text {; } \\
\text { Ambrosini et al., } 2009^{38}\end{array}$ & $\begin{array}{l}\text { Resultado } \\
\text { inconclusivo }\end{array}$ \\
\hline Tabagismo & $\begin{array}{l}\text { Padrão } \\
\text { Saudável }\end{array}$ & - & $\begin{array}{l}\text { Kourlaba et al., } \\
2009^{54}\end{array}$ & $\begin{array}{l}\text { Rodrigues et al., 2012 } \\
\text { Ambrosini et al., } 2009^{38}\end{array}$ & $\begin{array}{l}\text { Resultado } \\
\text { inconclusivo }\end{array}$ \\
\hline $\begin{array}{l}\text { Nível de } \\
\text { atividade } \\
\text { física }\end{array}$ & $\begin{array}{l}\text { Padrão } \\
\text { Ocidental }\end{array}$ & $\begin{array}{l}\text { McNaughton et } \\
\text { al., } 2008^{42}\end{array}$ & - & 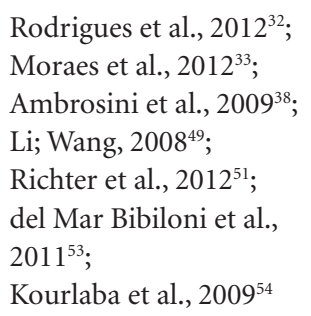 & Sem associação (C) \\
\hline $\begin{array}{l}\text { Nível de } \\
\text { atividade } \\
\text { física }\end{array}$ & $\begin{array}{l}\text { Padrão } \\
\text { Saudável }\end{array}$ & $\begin{array}{l}\text { Moraes et al., } \\
2012^{33} \text {; } \\
\text { Richter et al., } \\
2012^{51}\end{array}$ & - & $\begin{array}{l}\text { Ambrosini et al., } 2009^{38} \text {; } \\
\text { McNaughton et al., } \\
2008^{42} \text {; } \\
\text { Kourlaba et al., } 2009^{54}\end{array}$ & $\begin{array}{l}\text { Resultado } \\
\text { inconclusivo }\end{array}$ \\
\hline IMC elevado & $\begin{array}{l}\text { Padrão } \\
\text { Ocidental }\end{array}$ & $\begin{array}{l}\text { Salvatti et al., } \\
2011^{34} \text {; } \\
\text { Ambrosini et al., } \\
2010^{39} \text {; } \\
\text { Oddy et al., } \\
2013^{41} \text {; } \\
\text { Song et al., } 2010^{44} \text {; } \\
\text { Lozada et al., } \\
2007^{55}\end{array}$ & $\begin{array}{l}\text { Li; Wang, } 2008^{49} \text {; } \\
\text { Bibiloni et al., } \\
2011^{53}\end{array}$ & $\begin{array}{l}\text { Rodrigues et al., } 2012^{32} \text {; } \\
\text { Pinho et al., 2013 } \\
\text { Ambrosini et al., } 2009^{38} \text {; } \\
\text { McNaughton et al., } \\
2008^{42} \text {; } \\
\text { Garba et al., 2014 } \\
\text { Fitzgerald et al., 2013 } \\
\text { Richter et al., } 2012^{51}\end{array}$ & $\begin{array}{l}\text { Resultado } \\
\text { inconclusivo }\end{array}$ \\
\hline IMC elevado & $\begin{array}{l}\text { Padrão } \\
\text { Saudável }\end{array}$ & $\begin{array}{l}\text { Salvatti et al., } \\
2011^{34}\end{array}$ & $\begin{array}{l}\text { Pinho et al., } \\
2014^{36}\end{array}$ & 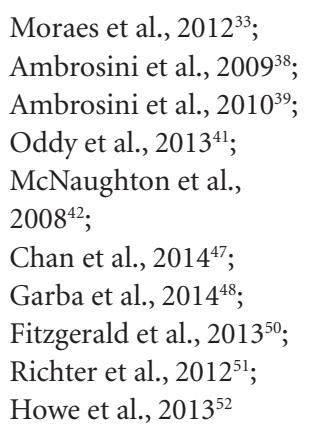 & Sem associação (C) \\
\hline CC & $\begin{array}{l}\text { Padrão } \\
\text { Ocidental }\end{array}$ & $\begin{array}{l}\text { Ambrosini et al., } \\
2010^{39}\end{array}$ & $\begin{array}{l}\text { Moraes et al., } \\
2012^{33}\end{array}$ & - & $\begin{array}{l}\text { Resultado } \\
\text { inconclusivo }\end{array}$ \\
\hline $\mathrm{CC}$ & $\begin{array}{l}\text { Padrão } \\
\text { Saudável }\end{array}$ & - & - & Ambrosini et al., $2010^{39}$ & Sem associação (D) \\
\hline Insulina & $\begin{array}{l}\text { Padrão } \\
\text { Ocidental }\end{array}$ & $\begin{array}{l}\text { Dishchekeni-an et } \\
\text { al., } 2011^{31}\end{array}$ & - & Ambrosini et al., $2010^{39}$ & $\begin{array}{l}\text { Resultado } \\
\text { inconclusivo }\end{array}$ \\
\hline Insulina & $\begin{array}{l}\text { Padrão } \\
\text { Saudável }\end{array}$ & - & - & Ambrosini et al., $2010^{39}$ & Sem associação (D) \\
\hline
\end{tabular}




\begin{tabular}{|c|c|c|c|c|c|}
\hline \multicolumn{6}{|c|}{ Quadro 2. continuação } \\
\hline $\begin{array}{l}\text { Fator } \\
\text { de risco }\end{array}$ & $\begin{array}{l}\text { Padrão } \\
\text { Alimentar }\end{array}$ & $\begin{array}{l}\text { Associação } \\
\text { positiva }\end{array}$ & $\begin{array}{l}\text { Associação } \\
\text { negativa }\end{array}$ & $\begin{array}{c}\text { Sem } \\
\text { associação }\end{array}$ & $\begin{array}{c}\text { Sumário da } \\
\text { evidência (GRADE) }\end{array}$ \\
\hline $\begin{array}{l}\text { Glicemia de } \\
\text { jejum }\end{array}$ & $\begin{array}{l}\text { Padrão } \\
\text { Ocidental }\end{array}$ & - & - & $\begin{array}{l}\text { Dishchekenian et al., } \\
2011^{31} \text {; } \\
\text { Ambrosini et al., } 2010^{39} \text {; } \\
\text { Ambrosini et al., } 2011^{40}\end{array}$ & Sem associação $(C)$ \\
\hline $\begin{array}{l}\text { Glicemia de } \\
\text { jejum }\end{array}$ & $\begin{array}{l}\text { Padrão } \\
\text { Saudável }\end{array}$ & - & $\begin{array}{l}\text { Ambrosini et al., } \\
2010^{39} \text {; } \\
\text { Ambrosini et al., } \\
2011^{40}\end{array}$ & - & $\begin{array}{l}\text { Associação negativa } \\
\text { (D) }\end{array}$ \\
\hline $\begin{array}{l}\text { Colesterol } \\
\text { total }\end{array}$ & $\begin{array}{l}\text { Padrão } \\
\text { Ocidental }\end{array}$ & $\begin{array}{l}\text { Dishchekeni-an et } \\
\text { al., } 2011^{31} \text {; } \\
\text { Ambrosini et al., } \\
2010^{39}\end{array}$ & - & Ambrosini et al., $2011^{40}$ & $\begin{array}{l}\text { Associação positiva } \\
\text { (D) }\end{array}$ \\
\hline $\begin{array}{l}\text { Colesterol } \\
\text { total }\end{array}$ & $\begin{array}{l}\text { Padrão } \\
\text { Saudável }\end{array}$ & - & - & $\begin{array}{l}\text { Ambrosini et al., } 2010^{39} \text {; } \\
\text { Ambrosini et al., } 2011^{40}\end{array}$ & Sem associação (D) \\
\hline LDL-c & $\begin{array}{l}\text { Padrão } \\
\text { Ocidental }\end{array}$ & $\begin{array}{l}\text { Dishchekeni-an et } \\
\text { al., } 2011^{31}\end{array}$ & - & Ambrosini et al., $2010^{39}$ & $\begin{array}{l}\text { Resultado } \\
\text { inconclusivo }\end{array}$ \\
\hline LDL_c & $\begin{array}{l}\text { Padrão } \\
\text { Saudável }\end{array}$ & - & - & Ambrosini et al., $2010^{39}$ & Sem associação (D) \\
\hline HDL-c & $\begin{array}{l}\text { Padrão } \\
\text { Ocidental }\end{array}$ & - & $\begin{array}{l}\text { Dishchekeni-an et } \\
\text { al., } 2011^{31}\end{array}$ & $\begin{array}{l}\text { Ambrosini et al., } 2010^{39} \text {; } \\
\text { Song et al., } 2010^{44}\end{array}$ & Sem associação (D) \\
\hline HDL-c & $\begin{array}{l}\text { Padrão } \\
\text { Saudável }\end{array}$ & $\begin{array}{l}\text { Ambrosini et al., } \\
2010^{39}\end{array}$ & - & - & $\begin{array}{l}\text { Associação positiva } \\
\text { (D) }\end{array}$ \\
\hline TG & $\begin{array}{l}\text { Padrão } \\
\text { Ocidental }\end{array}$ & Song et al., $2010^{44}$ & - & $\begin{array}{l}\text { Dishchekeni-an et al., } \\
2011^{31} \text {; } \\
\text { Ambrosini et al., 2010 } \\
\text { Ambrosini et al., } 2011^{40}\end{array}$ & Sem associação (D) \\
\hline TG & $\begin{array}{l}\text { Padrão } \\
\text { Saudável }\end{array}$ & - & - & $\begin{array}{l}\text { Ambrosini et al., } 2010^{39} \text {; } \\
\text { Ambrosini et al., } 2011^{40}\end{array}$ & Sem associação (D) \\
\hline PAS & $\begin{array}{l}\text { Padrão } \\
\text { Ocidental }\end{array}$ & $\begin{array}{l}\text { Dishchekeni-an et } \\
\text { al., } 2011^{31}\end{array}$ & - & $\begin{array}{l}\text { Ambrosini et al., } 2010^{39} \text {; } \\
\text { McNaughton et al., } 2008^{42}\end{array}$ & $\begin{array}{l}\text { Resultado } \\
\text { inconclusivo }\end{array}$ \\
\hline PAS & $\begin{array}{l}\text { Padrão } \\
\text { Saudável }\end{array}$ & - & - & Ambrosini et al., $2010^{39}$ & Sem associação (D) \\
\hline $\mathrm{PAD}$ & $\begin{array}{l}\text { Padrão } \\
\text { Ocidental }\end{array}$ & $\begin{array}{l}\text { Dishchekeni-an et } \\
\text { al., } 2011^{31}\end{array}$ & - & McNaughton et al., $2008^{42}$ & $\begin{array}{l}\text { Resultado } \\
\text { inconclusivo }\end{array}$ \\
\hline PAD & $\begin{array}{l}\text { Padrão } \\
\text { Saudável }\end{array}$ & - & $\begin{array}{l}\text { McNaughton et } \\
\text { al., } 2008^{42}\end{array}$ & - & $\begin{array}{l}\text { Associação negativa } \\
\text { (D) }\end{array}$ \\
\hline SM & $\begin{array}{l}\text { Padrão } \\
\text { Ocidental }\end{array}$ & $\begin{array}{l}\text { Ambrosini et al., } \\
2010^{39}\end{array}$ & - & - & $\begin{array}{l}\text { Associação positiva } \\
\text { (D) }\end{array}$ \\
\hline SM & $\begin{array}{l}\text { Padrão } \\
\text { Saudável }\end{array}$ & - & $\begin{array}{l}\text { Ambrosini et al., } \\
2010^{39}\end{array}$ & - & $\begin{array}{l}\text { Associação negativa } \\
\text { (D) }\end{array}$ \\
\hline
\end{tabular}

Legenda: CC - Circunferência da Cintura; HDL - Lipoproteína da Alta Densidade-c; IMC: Índice de Massa Corporal; LDL-c - Lipoproteína de Baixa Densidade-c; PAD - Pressão Arterial Diastólica; PAS- Pressão Arterial Sistólica; SM - Síndrome Metabólica; TG - Triglicerídeos. Qualidade da evidência (sistema GRADE): A - Alta; B - Moderada; C - Baixa; D - Muito Baixa. 
Padrões classificados como "Saudáveis" associaram-se positivamente com IMC elevado em um estudo ${ }^{34}$, em outro foi inversamente associado $^{36}$, e em dez não houve associação estatisticamente significativa ${ }^{33,38,39,41,42,47,48,50-52}$. Em um estudo observou-se associação positiva com HDL $-\mathrm{c}^{39}$. Com relação às alterações no metabolismo glicídico, dois estudos verificaram associação inversa com glicose sérica ${ }^{39,40}$. Associação inversa também foi verificada entre o padrão Saudável e a pressão arterial diastólica em adolescentes com idade $\geq 16$ anos em um estudo ${ }^{42}$ e com Síndrome Metabólica (SM) em outro estudo ${ }^{39}$.

O padrão Tradicional brasileiro foi associado positivamente com IMC elevado ${ }^{34}$ em um estudo, e em outro inversamente ${ }^{37}$. Com relação à associação com IMC eutrófico, verificou-se associação positiva em dois estudos ${ }^{32,34}$. O estudo brasileiro realizado exclusivamente com adolescentes com obesidade observou associação positiva entre o padrão Tradicional e glicemia, insulinemia e elevado triglicerídeo ${ }^{31}$, e inversa com HDL-c $\mathrm{c}^{31}$.

\section{Discussão}

Observou-se que quase todos os estudos incluídos na revisão, inclusive aqueles com brasilei$\operatorname{ros}^{31-34,36,37}$, registraram o padrão "Ocidental", caracterizados, no geral, por elevado consumo de produtos lácteos integrais, alimentos com elevado teor de açúcar simples e gordura, fast foods e refrigerantes, sendo esta constituição alimentar não saudável, obtida em adolescentes bem similar ao observado em pesquisas com adultos ${ }^{14,57}$.

Estes achados apontam que, na atualidade, o padrão estilo "Ocidental” constitui-se em um dos identificados na população adolescente com maior frequência, independentemente do país em estudo. Uma possível explicação para esta maior aderência aos padrões estilo "Ocidental" em detrimento dos considerados "Saudáveis" e/ ou "Tradicionais" de cada país, está na influência que a mídia exerce sobre o consumo de itens alimentares deste padrão, sendo comuns propagandas que objetivam levar os consumidores, notadamente os adolescentes, a preferirem lanches e alimentos de fácil preparo, em substituição às refeições que são características de padrões considerados como "Tradicional" e "Saudável"58,59.

Padrões classificados como Saudáveis foram obtidos por vários estudos incluídos nesta revisão. No geral, a constituição alimentar do padrão Saudável foi bem similar entre os estudos, os quais incluíram carnes brancas, cereais integrais e frutas, legumes e verduras (FLV) 33,34,36,38-42,50,51,54 Estes alimentos identificados como sendo do padrão Saudável condizem com os mais recomendados nos guias alimentares ${ }^{60}$.

Dois estudos incluídos na revisão obtiveram um padrão alimentar estilo "Mediterrâneo" 53,54, os quais são caracterizados, no geral, por reduzido consumo de carnes; moderado consumo de leite e derivados, principalmente os de baixo teor de gordura; elevada ingestão de azeite de oliva, frutas, hortaliças, leguminosas, cereais integrais e frutos secos; e de moderado a elevado consumo de peixes $^{18}$. A literatura tem demonstrado que o perfil dietético associado a este padrão alimentar caracteriza-se pelo seu elevado teor de gordura mono e poli-insaturadas, carboidratos complexos e fibras dietéticas, proteína vegetal e compostos antioxidantes ${ }^{18,61}$. Em virtude de seu bom perfil dietético e da sua inversa associação com diversas doenças crônicas, o padrão Mediterrâneo tem sido considerado como "Saudável"61. Sendo assim, este deve ser divulgado e a adesão ao mesmo incentivada entre adolescentes, público em que este padrão tem baixa aderência.

Os padrões alimentares "Tradicionais" de cada país apresentaram constituição alimentar bem divergente um dos outros, resultado da interação entre os aspectos culturais, econômicos, sociais e religiosos ${ }^{62}$. Em seis dos sete estudos incluídos na revisão que avaliaram padrões alimentares de adolescentes brasileiros ${ }^{31,32,34-37}$ foi obtido o "Tradicional", assim denominado em virtude de conter alimentos como feijão, arroz, carne vermelha, massas e gorduras, os quais são típicos na dieta do país ${ }^{63,64}$. O padrão alimentar "Tradicional" obtido no estudo realizado na Alemanha incluiu alimentos como carnes em geral (exceto galinha), batatas, pão branco, margarina, ovos, queijo, e peixe, ou seja, alimentos com elevado teor de gordura, isto é, apresentou constituição alimentar semelhante ao Tradicional obtido nos estudos brasileiros ${ }^{51}$. Na Coréia do Sul, o padrão "Tradicional" obtido incluiu arroz branco, kimchi e peixe ${ }^{43,44}$.

Devido a essa divergente constituição alimentar observada nos padrões Tradicionais de cada país, torna-se difícil realizar avaliações sistematizadas entre os padrões Tradicionais com os fatores de risco para DCV, tendo em vista obter o sumário da evidência, razão pela qual não o fizemos neste trabalho. Apenas para os padrões Saudável e Ocidental realizamos o sumário da evidência com classificação da qualidade segundo o sistema GRADE, principalmente porque estes são considerados um oposto ao outro. 
O tabagismo e o nível de atividade física são fatores de risco para DCV modificáveis que não são afetados diretamente pelos padrões alimentares, por mais que possa existir relação entre eles, pois quem busca ter hábitos de vida saudáveis o faz por meio da alimentação adequada, da prática de atividades físicas e não sendo fumante ${ }^{65,66}$. No presente estudo, o resultado da associação entre os padrões alimentares de adolescentes e o tabagismo foi inconclusivo. No que se refere à associação com a prática de atividade física, $o$ sumário da evidência para o padrão Ocidental revelou que não há associação significativa, com evidência de qualidade $\mathrm{C}$ - baixa, conforme o sistema GRADE. Já a associação com o padrão Saudável foi inconclusiva, segundo o mesmo sistema.

Salienta-se que a avaliação da prática regular de atividade física não é uma tarefa fácil, uma vez que são vários os instrumentos utilizados nela. Neste sentido, talvez os instrumentos utilizados nos estudos não apresentaram acurácia para identificar adequadamente uma associação.

Os estudos incluídos nesta revisão apresentaram várias associações entre padrão Ocidental e excesso de peso e alterações metabólicas. Porém, quando se realizou o sumário da evidência, observou-se que permaneceu a associação positiva apenas para colesterol total e SM, ambas com qualidade, segundo o sistema GRADE, D - muito baixa e, para nenhum dos desfechos, o padrão Ocidental foi considerado como de proteção. Já o padrão Saudável foi associado com resultados metabólicos satisfatórios, mesmo após realizar o sumário da evidência, como associação inversa com glicemia de jejum, Pressão Arterial Diastólica (PAD) e SM e positiva com HDL-c, e para nenhum dos desfechos foi considerado como de risco. A qualidade da evidência para estas associações também foi $\mathrm{D}$ - muito baixa.

Por mais que a qualidade da evidência dos achados deste estudo tenha sido baixa e muito baixa, observou-se que estão de acordo com o verificado em outros com adolescentes, de modo que o consumo de alimentos do padrão Ocidental com elevado teor energético, de gordura total, gordura saturada e carboidrato simples foi associado a alguns fatores de risco para DCV, enquanto que o consumo de alimentos do padrão Saudável, os quais são geralmente ricos em carboidratos complexos, fibra, gorduras monoinsaturadas e poliinsaturadas, vitaminas e minerais foi inversamente associado ${ }^{67,68}$.

Considerando a dieta como um fator de risco cardiovascular modificável, medidas de educação alimentar e nutricional são necessárias, tendo em vista auxiliar na prevenção e no controle das doenças cardiovasculares para assegurar o pleno potencial de crescimento e desenvolvimento dos adolescentes ${ }^{69}$.

Este artigo de revisão apresenta algumas limitações como a possível não inclusão de todos os artigos referentes aos padrões alimentares de adolescentes identificados por métodos a posteriori, considerando que não foram utilizadas todas as bases de dados. Porém, salienta-se que foram empregadas várias combinações de termos em bases de dados relevantes objetivando obter o maior número possível de estudos, assim como a realização da busca manual que incorporou mais registros. A qualidade da evidência dos estudos incluídos nessa revisão foi baixa e muito baixa, em virtude, principalmente, do delineamento observacional e de vários estudos apresentarem limitações metodológicas que comprometeram a qualidade da evidência. Entretanto, salienta-se a importância dos achados do presente estudo em virtude de o mesmo ser inédito, ressaltando a necessidade de mais pesquisas com delineamentos apropriados. Ademais, há que se considerar as limitações próprias ao uso de padrões alimentares em estudos de epidemiologia nutricional, haja vista as dificuldades em se conhecer verdadeiramente o real consumo do indivíduo, devido aos erros sistemáticos e aleatórios envolvidos nas várias etapas até a derivação dos mesmos.

\section{Conclusão}

O presente estudo observou que na população adolescente das mais diversas regiões do mundo o principal padrão alimentar identificado foi o "Ocidental". Isto é preocupante, uma vez que este foi associado positivamente com fatores de risco para DCV como colesterol total e SM, enquanto que o padrão Saudável foi associado com resultados metabólicos satisfatórios, como associação inversa com glicemia de jejum, PAD e SM e positiva com HDL-c. Por mais que estes resultados devam ser analisados com prudência, uma vez que a qualidade da evidência dos estudos incluídos foi baixa ou muito baixa, sendo necessários estudos com maior rigor metodológico, observa-se a necessidade de medidas que visem à promoção da alimentação saudável em adolescentes, tendo em vista proporcionar o pleno desenvolvimento e crescimento dos mesmos, bem como auxiliar na prevenção e/ou controle dos fatores de risco para DCV nesta população. 


\section{Colaboradores}

DFO Silva e SCVC Lima contribuíram na concepção do artigo, coleta, análise e interpretação dos dados, redação e revisão crítica do texto e aprovação da versão final para publicação. CO Lyra contribuiu na análise e interpretação dos dados, redação e revisão crítica do texto e aprovação da versão final para publicação.

\section{Referências}

1. Barkoukis H. Importance of understanding food consumption patterns. J Am Diet Assoc 2007; 107(2):234236.

2. Michels KB, Schulze MB. Can dietary patterns help us detect diet-disease associations? Nutr Res Rev 2005; 18(2):241-248.

3. Slattery ML. Analysis of dietary patterns in epidemiological research. Appl Physiol Nutr Metab 2010; 35(2):207-210.

4. Hu FB. Dietary pattern analysis: a new direction in nutritional epidemiology. Curr Opin Lipidol 2002; 13(1): 3-9.

5. Fisberg RM, Marchioni ML, Colucci ACA. Avaliação do consumo alimentar e da ingestão de nutrientes na prática clínica. Arq Bras Endocrinol Metab 2009; 53(5):617624.

6. Willet WC, editor. Nutritional Epidemiology. $2^{\text {nd }}$ ed. New York: Oxford University Press; 1998.

7. Panagiotakos D. $\alpha$-priori versus $\alpha$-posterior methods in dietary pattern analysis: a review in nutrition epidemiology. Nutr Bull 2008; 33(4):311-315.

8. Panagiotakos DB, Pitsavos C, Stefanadis C. $\alpha$-Priori and $\alpha$-Posterior Dietary Pattern Analyses Have Similar Estimating and Discriminating Ability in Predicting 5-Y Incidence of Cardiovascular Disease: Methodological Issues in Nutrition Assessment. J Food Sci 2009; 74(7):H218-H224.

9. Schulze MB, Hoffmann K. Methodological approaches to study dietary patterns in relation to risk of coronary heart disease and stroke. Br J Nutr 2006; 95(5):860-869.

10. Hearty AP, Gibney MJ. Comparison of cluster and principal component analysis techniques to derive dietary patterns in Irish adults. Br J Nutr 2009; 101(4):598-608.

11. Cunha DB, de Almeida RM, Sichieri R, Pereira RA. Association of dietary patterns with BMI and waist circumference in a low-income neighbourhood in Brazil. Br J Nutr 2010; 104(6):908-913.

12. Marchioni DML, Latorre MDRDD, Eluf-Neto J, Wünsch-Filho V, Fisberg RM. Identification of dietary patterns using factor analysis in an epidemiological study in São Paulo. Sao Paulo Med J 2005; 123(3):124127.

13. Flores M, Macias N, Rivera M, Lozada A, Barquera S, Rivera-Dommarco J, Tucker KL. Dietary patterns in Mexican adults are associated with risk of being overweight or obese. J Nutr 2010; 140(10):1869-1873.

14. James D. Cluster analysis defines distinct dietary patterns for African-American men and women. J Am Diet Assoc 2009; 109(2):255-262.

15. Hoffman K, Schulze MB, Schienkiewitz A, Nöthlings U, Boeing $\mathrm{H}$. Application of a new statistical method to derive dietary patterns in nutritional epidemiology. Am J Epidemiol 2004; 159(10):935-944.

16. Sherafat-Kazemzade R, Egtesadi S, Mirmiran P, Gohari M, Farahani SJ, Esfahani FH, Vafa MR, Hedayati M, Azizi F. Dietary patterns by reduced rank regression predicting changes in obesity indices in a cohort study: Tehran lipid and glucose study. Asia Pac J Clin Nutr 2010; 19(1):22-32. 
17. Okubo H, Sasaki S, Murakami K, Kim MK, Takahashi Y, Hosoi Y, Itabashi M; Freshmen in Dietetic Courses Study II Group. Dietary patterns associated with functional constipation among Japanese women aged 18 to 20 years: a cross-sectional study. J Nutr Sci Vitaminol (Tokyo) 2007; 53(3):232-238.

18. Rumawas ME, Meigs JB, Dwyer JT, McKeown NM, Jacques PF. Mediterranean-style dietary pattern, reduced risk of metabolic syndrome traits, and incidence in the Framingham Offspring Cohort. Am J Clin Nutr 2009; 90(6):1608-1614.

19. Neutzling MB, Araújo CLP, Vieira MFA, Hallal PC, Menezes AMB. Freqüência de consumo de dietas ricas em gordura e pobres em fibra entre adolescentes. Rev Saude Publica 2007; 41(3):336-342.

20. Enes CC, Pegolo GE, Silva MVD. Influência do consumo alimentar e do padrão de atividade física sobre o estado nutricional de adolescentes de Piedade, São Paulo. Rev paul pediatr 2009; 27(3):265-271.

21. Cardoso LDO, Alves LC, Castro IRRD, Leite IDC, Machado CJ. Use of the Grade of Membership method to identify consumption patterns and eating behaviors among adolescents in Rio de Janeiro, Brazil. Cad Saude Publica 2011; 27(2):335-346.

22. Diethelm K, Jankovic N, Moreno LA, Huybrechts I, De Henauw S, De Vriendt T, González-Gross M, Leclercq C, Gottrand F, Gilbert CC, Dallongeville J, CuencaGarcia M, Manios Y, Kafatos A, Plada M, Kersting M; HELENA Study Group. Food intake of European adolescents in the light of different food-based dietary guidelines: results of the HELENA (Healthy Lifestyle in Europe by Nutrition in Adolescence) Study. Public Health Nutr 2012; 15(3):386-398.

23. Maynard M, Gunnell D, Emmett P, Frankel S, Smith GD. Fruit, vegetables, and antioxidants in childhood and risk of adult cancer: the Boyd Orr cohort. J Epidemiol Community Health 2003; 57(3):218-225.

24. Malik VS, Fung TT, van Dam RM, Rimm EB, Rosner B, $\mathrm{Hu}$ FB. Dietary patterns during adolescence and risk of type 2 diabetes in middle-aged women. Diabetes care 2012; 35(1):12-18.

25. de Henauw S, Gottrand F, De Bourdeaudhuij I, Gonzalez-Gross M, Leclercq C, Kafatos A, Molnar D, Marcos A, Castillo M, Dallongeville J, Gilbert CC, Bergman P, Widhalm K, Manios Y, Breidenassel C, Kersting M, Moreno LA; HELENA Study Group. Nutritional status and lifestyles of adolescents from a public health perspective. The HELENA Project-Healthy Lifestyle in Europe by Nutrition in Adolescence. J Publ Health 2007; 15(3):187-197.

26. Moher D, Liberati A, Tetzlaff J, Altman DG. Preferred reporting items for systematic reviews and meta-analyses: the PRISMA statement. PLoS Med 2009; 6:e1000097.

27. von Elm E, Altman DG, Egger M, Pocock SJ, Gotzsche PC, Vandenbroucke JP. The Strengthening the Reporting of Observational Studies in Epidemiology (STROBE) statement: guidelines for reporting observational studies. J Clin Epidemiol 2008; 61(4):344-349.

28. Guyatt G, Vist G, Falck-Ytter Y, Kunz R, Magrini N, Schunemann H. An emerging consensus on grading recommendations? Evidence-Based Medicine 2006; 11(1):2-4.
29. Guyatt GH, Oxman AD, Vist GE, Kunz R, Falck-Ytter Y, Alonso-Coello P, Schünemann HJ; GRADE Working Group. GRADE: an emerging consensus on rating quality of evidence and strength of recommendations. BMJ (Clinical Research Ed.) 2008; 336(7650):924-926.

30. Brasil. Ministério da Saúde (MS). Secretaria de Atenção à Saúde. Departamento de Atenção Básica. Cadernos de Atenção Básica. Estratégias para o cuidado da pessoa com doença crônica: diabetes mellitus. Brasília: MS; 2013.

31. Dishchekenian VR, Escrivão MA, Palma D, Ancona -Lopez F, Araújo EA, Taddei JA. Padrões alimentares de adolescentes obesos e diferentes repercussões metabólicas. Rev Nutr 2011; 24(1):17-29.

32. Rodrigues PRM, Pereira RA, Cunha DB, Sichieri R, Ferreira MG, Vilela AAF. Fatores associados a padrões alimentares em adolescentes: Um estudo de base escolar em Cuiabá, Mato Grosso. Rev Bras Epidemiol 2012; 15(3):662-674.

33. Moraes ACF, Adami F, Falcão MC. Understanding the correlates of adolescents' dietary intake patterns. A multivariate analysis. Appetite 2012; 58(3):1057-1062.

34. Salvatti AG, Escrivão MAMS, Taddei JAAC. Padrões alimentares de adolescentes na cidade de São Paulo. Rev Nutr 2011; 24(5):703-713.

35. de Morais CMM, Pinheiro LGB, Lima SCVC, Lyra CO, de Sena Evangelista KCM, Lima KC, Pedrosa LF. Dietary patterns of young adolescents in urban areas of Northeast Brazil. Nutr Hosp 2013; 28(6):1977-1984.

36. Pinho LD, Silveira MF, Botelho ACDC, Caldeira AP. Identification of dietary patterns of adolescents attending public schools. J Pediatr (Rio J) 2014; 90(3):267272.

37. Borges CA, Enes CC, Slater B, Conde WL. BMI Changes Associated With Dietary Trends Among Brazilian Adolescents. ICAN 2012 4(6):361-368.

38. Ambrosini GL, Oddy WH, Robinson M, O’Sullivan TA, Hands BP, de Klerk NH, Silburn SR, Zubrick SR, Kendall GE, Stanley FJ, Beilin LJ. Adolescent dietary patterns are associated with lifestyle and family psycho-social factors. Public Health Nutr 2009; 12(10):1807-1815.

39. Ambrosini GL, Huang RC, Mori TA, Hands BP, O'Sullivan TA, de Klerk NH, Beilin LJ, Oddy WH. Dietary patterns and markers for the metabolic syndrome in Australian adolescents. Nutr Metab Cardiovasc Dis 2010; 20(4):274-283.

40. Ambrosini GL, O'Sullivan TA, de Klerk NH, Mori TA, Beilin LJ, Oddy WH. Relative validity of adolescent dietary patterns: a comparison of a FFQ and $3 \mathrm{~d}$ food record. Br J Nutr 2011; 105:625.

41. Oddy WH, Herbison CE, Jacoby P, Ambrosini GL, O'Sullivan TA, Ayonrinde OT, Olynyk JK, Black LJ, Beilin LJ, Mori TA, Hands BP, Adams LA. The Western Dietary Pattern Is Prospectively Associated With Nonalcoholic Fatty Liver Disease in Adolescence. Am J Gastroenterol 2013; 108(5):778-785.

42. McNaughton SA, Ball K, Mishra GD, Crawford DA. Dietary patterns of adolescents and risk of obesity and hypertension. J Nutr 2008; 138(2):364-370.

43. Song Y, Joung H, Engelhardt K, Sun YY, Paik HY. Traditional vs modified dietary patterns and their influence on adolescents nutritional profile. Br J Nutr 2005; 93:943. 
44. Song Y, Park MJ, Paik HY, Joung H. Secular trends in dietary patterns and obesity-related risk factors in Korean adolescents aged 10-19 years. Int J Obes 2010; 34(1):48-56.

45. Joung H, Hong S, Song Y, Ahn BC, Park MJ. Dietary patterns and metabolic syndrome risk factors among adolescents. Korean J Pediatr 2012; 55(4):128-135.

46. Weng TT, Hao JH, Quian QW, Caco H, Fu JL, Sun Y, Huang L, Tao FB. Is there any relationship between dietary patterns and depression and anxiety in Chinese adolescents? Public Health Nutr 2011; 15(4):673-682.

47. Chan R, Chan D, Lau W, Lo D, Li L, Woo J. A Cross-sectional Study to Examine the Association Between Dietary Patterns and Risk of Overweight and Obesity in Hong Kong Chinese Adolescents Aged 10-12 Years. J Am Coll Nutr 2014; 33(6):450-458.

48. Garba JA, Rampal L, Hejar AR, Salmiah MS. Major Dietary Patterns and their Associations with Socio-demographic Characteristics and Obesity among Adolescents in Petaling District, Malaysia. Malays J Med Heal Sci 2014; 10(1):13-21.

49. Li J, Wang Y. Tracking of dietary intake patterns is associated with baseline characteristics of urban low-income African-American adolescents. I Nutr 2008; 138(1):94-100.

50. Fitzgerald A, Heary C, Kelly C, Nixon E, Shevlin M. Self-efficacy for healthy eating and peer support for unhealthy eating are associated with adolescents' food intake patterns. Appetite 2013; 63:48-58.

51. Richter A, Heidemann C, Schulze MB, Roosen J, Thiele $S$, Mensink GB. Dietary patterns of adolescents in Germany-Associations with nutrient intake and other health related lifestyle characteristics. BMC pediatr 2012; $12: 35$

52. Howe AS, Black KE, Wong JE, Parnell WR, Skidmore PM. Dieting status influences associations between dietary patterns and body composition in adolescents: a cross-sectional study. Nutr J 2013; 12:51.

53. del Mar Bibiloni M, Martínez E, Llull R, Pons A, Tur JA. Western and Mediterranean dietary patterns among Balearic Islands' adolescents: socio-economic and lifestyle determinants. Public Health Nutr 2011; 15(4):683692.

54. Kourlaba G, Panagiotakos DB, Mihas K, Alevizos A, Marayiannis K, Mariolis A, Tountas Y. Dietary patterns in relation to socio-economic and lifestyle characteristics among Greek adolescents: a multivariate analysis. Public Health Nutr 2009; 12(9):1366-1372.

55. Lozada AL, Flores M, Rodrígues S, Barquera S. Patrones dietarios en adolescentes mexicanas. Una comparación de dos métodos. Encuesta Nacional de Nutrición, 1999. Salud Publica Mex 2007; 49(4):263-273.

56. Ochoa-Avilés A, Verstraeten R, Lachat C, Andrade S, Van Camp J, Donoso S, Kolsteren P. Dietary intake practices associated with cardiovascular risk in urban and rural Ecuadorian adolescents: a cross-sectional study. BMC public health 2014; 14:939.

57. de Oliveira EP, McLellan KCP, de Arruda Silveira LV, Burini RC. Dietary factors associated with metabolic syndrome in Brazilian adults. Nutr J 2012; 11:13.

58. Almeida SS, Nascimento PC, Quaioti TC. Quantidade e qualidade de produtos alimentícios anunciados na televisão brasileira. Rev Saude Publica 2002; 36(3):353-355.
59. Rossi CE, Albernaz DO, Vasconcelos FDAGD, Assis MAAD, Di Pietro PF. Influência da televisão no consumo alimentar e na obesidade em crianças e adolescentes: uma revisão sistemática. Rev Nutr 2010; 23(4):607620.

60. Brasil. Ministério da Saúde (MS). Secretaria de Atenção à Saúde. Coordenação Geral de Alimentação e Nutrição. Guia alimentar para a população brasileira. Brasília: MS; 2014.

61. Serra-Majem L, Roman B, Estruch R. Scientific evidence of interventions using the Mediterranean diet: a systematic review. Nutr Rev 2006; 64(2 Pt 2):S27-S47.

62. Kittler PG, Sucher KP. Food and Culture. $4^{\text {th }}$. Belmont Wadsworth; 2004.

63. Neumann AICP, Martins IS, Marcopito LF, Araujo EAC. Padrões alimentares associados a fatores de risco para doenças cardiovasculares entre residentes de um município brasileiro. Rev Panam Salud Publica 2007; 22(5):329-339.

64. Nascimento S, Barbosa FS, Sichieri R, Pereira RA. Dietary availability patterns of the brazilian macro-regions. Nutr J 2011; 10:79.

65. Beck CC, Lopes ADS, Giuliano IDCB, Borgatto AF. Fatores de risco cardiovascular em adolescentes de município do sul do Brasil: prevalência e associações com variáveis sociodemográficas. Rev Bras Epidemiol 2011; 14(1):36-49.

66. Pearson N, Atkin AJ, Biddle SJS, Gorely T, Edwardson C. Patterns of adolescent physical activity and dietary behaviours. Int J Behav Nutr Phys Act 2009; 6:7.

67. Romero-Polvo A, Denova-Gutiérrez E, Rivera-Paredez B, Castañón S, Gallegos-Carrillo K, Halley-Castillo E, Borges G, Flores M, Salmerón J. Association between dietary patterns and insulin resistance in Mexican children and adolescents. Ann Nutr Metab 2012; 61(2):142150

68. Alcântara Neto ODD, Silva RDCR, Assis AMO, Pinto EDJ. Factors associated with dyslipidemia in children and adolescents enrolled in public schools of Salvador, Bahia. Rev Bras Epidemiol 2012; 15(2):335-345.

69. dos Santos MG, Pegoraro M, Sandrini F, Macuco EC Fatores de risco no desenvolvimento da aterosclerose na infância e adolescência. Arq Bras Cardiol 2008; 90(4):301-308.

Artigo apresentado em 04/04/2014

Aprovado em 22/10/2015

Versão final apresentada em 24/10/2015 
